
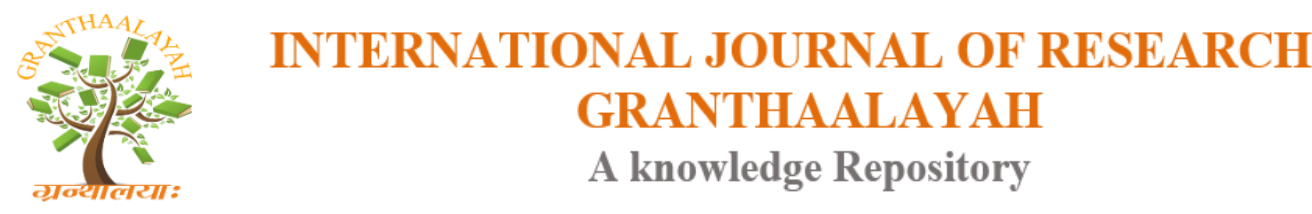

Science

\title{
APPLICATIONS OF ARTIFICIAL INTELLIGENCE IN HUMAN LIFE
}

\author{
Rupali Kamble $^{* 1}$, Prof. Deepali Shah ${ }^{2}$ \\ ${ }^{*}$ PG Student, Dept. of MCA, NCRD’s SIMS, Navi Mumbai, India \\ ${ }^{2}$ Assistant Professor, Department of MCA, NCRD’s SIMS, Navi Mumbai, India
}

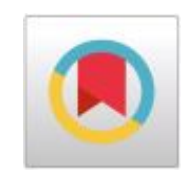

\begin{abstract}
Artificial Intelligence (AI) has revolutionized in information technology.AI is a subfield of computer science that includes the creation of intelligent machines and software that work and react like human beings. AI and its Applications gets used in various fields of life of humans as expert system solve the complex problems in various areas as science, engineering, business, medicine, video games and Advertising. But "Do any traffic lights use Artificial Intelligence??", I thought a lot of this when waiting in a red light.

This paper gives an overview of Artificial Intelligence and its applications used in human life. This will explore the current use of Artificial Intelligence technologies in Network Intrusion for protecting computer and communication networks from intruders, in the medical area-medicine, to improve hospital inpatient care, for medical image classification, in the accounting databases to mitigate the problems of it, in the computer games, and in Advertising. Also, it will show artificial intelligence principle and how they were applying in traffic signal control, how they solve some traffic problem in actual. This paper gives an introduction to a self-learning system based on RBF neural network and how the system can simulate the traffic police's experience. This paper is focusing on how to evaluate the effect of the control with the changing of the traffic and adjust the signal with the different techniques of Artificial Intelligence.
\end{abstract}

Keywords: Artificial Intelligence; Intrusion Detection Systems; Neural Networks (Computer); Expert System; Self-Learning System; RBF Neural Network.

Cite This Article: Rupali Kamble, and Prof. Deepali Shah. (2018). "APPLICATIONS OF ARTIFICIAL INTELLIGENCE IN HUMAN LIFE." International Journal of Research - Granthaalayah, 6(6), 178-188. https://doi.org/10.29121/granthaalayah.v6.i6.2018.1363.

\section{Introduction}

Artificial intelligence was coming into existence1956. It has rapid progress in more than 50 years. AI sense machines will improve human abilities in numerous zones. As It is claimed that artificial intelligence is apply very widely in the research of computer science and operational research areas. Intelligence is commonly considered as the ability to collect knowledge and reason about knowledge to solve complex problems. In the near Future intelligent machines will replace human capabilities in many areas. John McCarthy coined the term in 1956 as branch of computer science 
concerned with making computers behave like humans. It is the study of the computation that makes it possible to perceive reason and act. Artificial intelligence has the sequence of arena as, Intelligence $=$ perceive + Analyses + React, is different from psychology because of its significance on computation and is different from computer science because of its significance on perception, reasoning and action. It makes machines smarter and more useful. It works with the help of artificial neurons (artificial neural network) and scientific theorems (if then statements and logics). Computer is having the ability which imitate the man intelligence action. Artificial Intelligence is the study of mental faculties through the use of computational models. Artificial Intelligence is playing an important role in understanding and performing intelligent tasks such as reasoning, learning new skills and adopting to new situations and problems. The different distinctive methods applied in artificial intelligence are Neural Network, Fuzzy Logic, Evolutionary Computing, and Hybrid Artificial Intelligence.

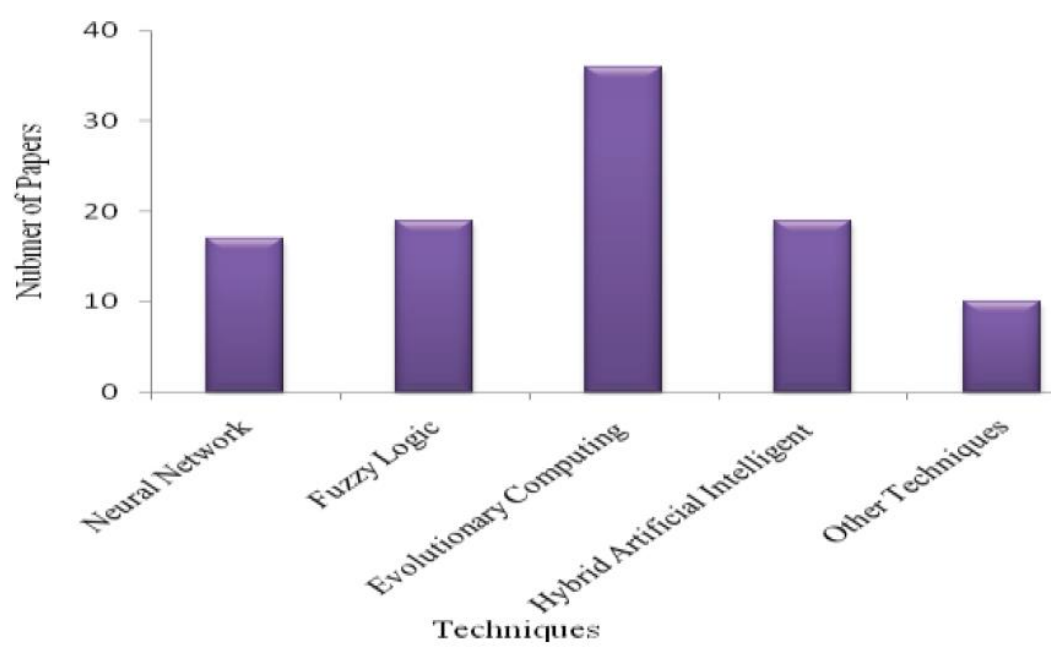

Figure 1: Papers published on different Artificial Intelligence Techniques used

Artificial Intelligence insight has incredibly enhanced the execution of the assembling and administration frameworks of human mind and machines, it has the advantages over the natural intelligence as it is more permanent, consistent, less expensive, proficiency has the ease of duplication and dissemination, can be documented and can perform certain tasks much faster and better than the human.

Intelligent technologies which are penetrating to different parts of human life don't ignore transportation. As an example, we can take intelligent transport systems and automated transport systems which use information, transportation and communication technologies implemented to vehicles or to infrastructure. These systems aim to increase people or goods mobility along with increasing road safety and transportation comfort, reduction of transport collisions and impacts on environment.

\section{Literature Review}

Artificial intelligence refers to the study and developments of intelligent machines and software that are performing intelligent task like human beings and can reason, learn, gather knowledge, communicate, manipulate and perceive the objects. AI technologies have matured to the point in 
offering real practical benefits in many of their applications. Major Artificial Intelligence areas are Expert Systems, to take care of the unpredictable issues in different regions of Science and technologies, Natural Language Processing, Speech Understanding, Robotics and Sensory Systems, Computer Vision and Scene Recognition, Intelligent Computer- Aided Instruction, Neural Computing, architectural, finance, business enterprises, software and gaming industries, Aero spacing, drug, climate anticipating etc.. Application areas of Artificial Intelligence is vastly affecting the different fields of life as a specialist expert system is a rapidly growing technology which is having a huge impact on various fields of life.

\section{Problem Definition}

Besides AI, I thought a lot of this when waiting in a red light with more than 3 cars and no car is passing on the green, that's one of many problems. No traffic lights use artificial intelligence. In any case, I can see that there are some great uses of AI to improve activity by decreasing crossing points mishaps, lessening movement in a few spots. The great old clock on a red light is never again a smart thought in a few crossing points, in truth at times police keeps an eye on control the lights to lessen the activity, something that should be possible adequately by an AI. There are an ever-increasing number of individuals utilizing autos regular, and more mischances on convergences, in actuality $40 \%$ of mishaps happen in convergences yet not all crossing points have movement lights. So, it is an incredible plan to utilize AI in rush hour gridlock lights.

\section{Objective}

The objective of this study is to Control the actual traffic condition and improve the passing ability of intersection. A self-learning system based on RBF neural network can simulate the traffic police's experience. According to the queue length in each intersection, the system can give out both the signal cycle and the spilt of intersection. Furthermore, it can evaluate the effect of the control with the changing of the traffic and adjust the signal. Here main purpose of this study is to Control the traffic and accidents on intersections.

\section{Scope}

Artificial Intelligence having different techniques like Neural Network, Fuzzy Logic, Evolutionary Computing, and Hybrid Artificial Intelligence which were used in different applications of AI. The different types of neural network can help in making traffic better.

\section{Research Methodology}

\subsection{Areas of Artificial Intelligence}

\subsubsection{Language Understanding}

The ability to "understand" and respond to the natural language. To translate from spoken language to a written form and to translate from one natural language to another natural language.

- Speech Understanding

- Semantic Information Processing (Computational Linguistics) 
- Question Answering

- Information Retrieval

- Language Translation

\subsubsection{Learning and Adaptive Systems}

The ability to adapt behavior based on previous experience, and to develop general rules concerning the world based on such experience.

- Cybernetics

- Concept Formation

\subsubsection{Problem Solving}

Ability to formulate a problem in a suitable representation, to plan for its solution and to know when new information is needed and how to obtain it.

- Inference (Resolution-Based Theorem Proving, Plausible Inference and Inductive Inference)

- Interactive Problem Solving

- Automatic Program Writing

- Heuristic Search

\subsubsection{Perception (Visual)}

The ability to analyze a sensed scene by relating it to an internal model which represents the perceiving organism's "knowledge of the world." The result of this analysis is a structured set of relationships between entities in the scene.

- Pattern Recognition

- Scene Analysis

\subsubsection{Modeling}

The ability to develop an internal representation and set of transformation rules which can be used to predict the behavior and relationship between some set of real-world objects or entities.

- The Representation Problem for Problem Solving Systems

- Modeling Natural Systems (Economic, Sociological, Ecological, Biological etc.)

- Hobot World Modeling (Perceptual and Functional Representations)

\subsubsection{Robots}

A combination of most or all the above abilities with the ability to move over and manipulate objects.

- Exploration

- Transportation/Navigation

- Industrial Automation (e.g., Process Control, Assembly Tasks, Executive Tasks)

- Security 
- Other (Agriculture, Fishing, Mining, Sanitation, Construction, etc.)

- Military

- Household

\subsubsection{Games}

The ability to accept a formal set of rules for games such as Chess, Video games, Go etc., and to translate these rules into a representation which allows problem-solving and learning abilities to be used in reaching an adequate level of performance and competing at the highest level in strategic game systems.

- Particular Games (Chess, Go, Bridge, etc.)

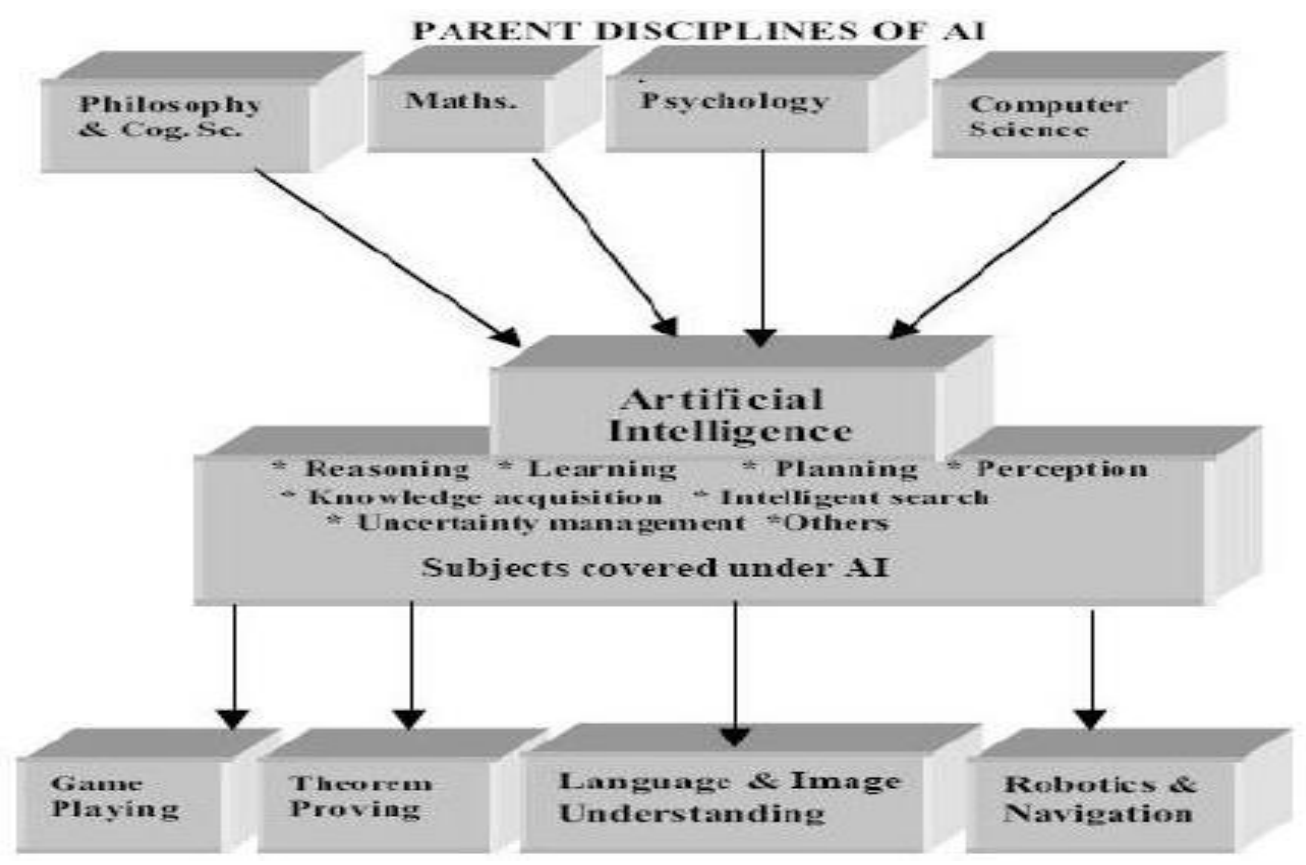

Figure 2: Areas of Artificial Intelligence

\subsection{Applications of Artificial Intelligence}

\subsubsection{Application of Artificial Intelligence Techniques in Network Intrusion Detection}

Intrusion Detection Systems (IDS) utilizes the different Artificial Intelligence methods for shielding PC and correspondence systems from gatecrashers. Interruption Detection System (IDS) is the way toward observing the occasions happening in system and identifying the indications of interruption

- Artificial Neural Network in IDS: ANN is a scientific model that comprises of an interconnected gathering of counterfeit neurons which forms the data. In IDS ANN are utilized to display complex connections amongst information sources and yields or to discover designs in information. In this a neuron figures the entirety by increasing contribution by weight and applies an edge. The outcome is transmitted to resulting 
neurons. Essentially, the ANN has been summed up to: $y i=f(\sigma w i k x k+\mu i)(1) k$ Where wik are weights appended to the sources of info, $x k$ are contributions to the neuron $I, \mu \mathrm{i}$ is a limit, $f(\bullet)$ is an exchange capacity and yi is the yield of the neuron.

- Fuzzy Inference Systems (FIS) in IDS: Sampada et al proposed two machine learning standards: Artificial Neural Networks and Fuzzy Inference System, for the outline of an Intrusion Detection System. They utilized SNORT to perform ongoing activity examination and parcel signing on IP arrange amid the preparation period of the framework. They built a mark design database utilizing Protocol Analysis and NeuroFuzzy learning technique. They at that point tried and approved the models utilizing the 1998 DARPA Intrusion Detection Evaluation Data and TCP dump crude information. The informational collection contains 24 assault composes. The assaults fall into four principle classes viz. Foreswearing of Service (DOS), Remote to User (R2L), User to Root (U2R), and Probing. From the outcomes, it was demonstrated that the Fuzzy Inference System was quicker in preparing, taking couple of moments, than the Artificial Neural Networks which took couple of minutes to join. For the most part, the two methods ended up being great, however with the Fuzzy Inference System having an edge over Artificial Neural Networks with its higher order exactness's. Their trial additionally demonstrated the significance of variable choice, as the two procedures performed more terrible when every one of the factors were utilized without determination of the factors. Great outcomes were recorded when a subset (around 40\%) of the factors were utilized.

\subsubsection{Application of Artificial Intelligence Techniques in Medical Area}

Artificial knowledge methods can possibly be connected in relatively every field of restorative zone.

\section{- Artificial Intelligence in Medicine}

(a) Fuzzy Expert Systems in Medicine: Fuzzy logic is a data handling methodology that permits ambiguity and hence is particularly suited to medical applications. It captures and implements the concept of fuzziness in a computationally effective manner. The most likely area of application for this theory used in medical diagnostics and, to a lesser extent, in the description of biological systems. Fuzzy expert systems use the structure of a series of, if - then ${ }^{\text {ee }}$ rules for modeling.

\begin{tabular}{|ll|}
\hline IF & Change in bowel habit OR Rectal bleeding \\
THEN & Consult your doctor \\
\hline
\end{tabular}

Figure 3: A typical fuzzy rule system.

The techniques of fuzzy logic have been explored in many medical applications. Fuzzy logic is preferred over the multiple logistic regression analysis in diagnosing lung cancer using tumor marker profiles. Fuzzy logic is also used in the diagnosis of acute leukemia and breast and pancreatic cancer and also predict patients ${ }^{\text {ee }}$ survival with breast cancer. They can also characterize MRI images of brain tumors, ultrasound images of the breast, ultrasound. Fuzzy logic controllers have been designed for the administration of vacillators in the preoperative period to control blood pressure. 
- Evolutionary Computation in Medicine: Evolutionary calculation is the general term for a few computational strategies in view of common advancement process that mimics the system of common choice and survival of the fittest in taking care of certifiable issues. The most broadly utilized type of developmental calculation for restorative applications are "Genetic Algorithms". Hereditary Algorithms" in light of the characteristic natural advancement are the most generally utilized type of developmental calculation for restorative applications. The standards of Hereditary calculations have been utilized to anticipate result in basically sick patients. X-ray division of mind tumors to gauge the viability of treatment procedures is additionally done through transforrmative calculation. They have additionally been utilized as a part of modernized examination of mammographic miniaturized scale calcification.

(a) Using Artificial Intelligence to Improve Hospital Inpatient Care: Clinical choice emotionally supportive networks (CDSS) were one of the main effective applications of AI, concentrating Primarily on the analysis of a patient's condition given his indications and statistic data. Mycin a lead-based master framework for recognizing microscopic organisms making contaminations and suggesting anti toxins treat these contaminations was created in 1970 under crafted by CDSS for medicinal finding. Pathfinder, which utilized Bayesian systems to help pathologists more precisely analyze lymph-hub illnesses. AI has likewise been valuable for PC supported location of tumors in therapeutic pictures. Such methodologies help in the conclusion of different types of growth, and inborn heart surrenders.

(b) Artificial Intelligence Approaches for Medical Image Classification: Artificial intelligence techniques are used for diagnostic sciences in biomedical image classification. Model-based intelligent analysis a decision-support tools are important in medical imaging for computer-assisted diagnosis and evaluation. CAD helps radiologist who uses the output from a computerized analysis of medical images as a second opinion in detecting lesions, assessing extent of disease, and improving the accuracy and consistency of radiological diagnosis to reduce the rate of false negative cases.

(c) Artificial Neural Networks Approach on Diagnostic Science: The accompanying subsections will talk about how ANN is used for picture grouping over ages.

(d) Endoscopic Images: Image classification is an important step in CAD. In classification of endoscopic images, a hybrid implementation by advanced fuzzy inference neural network which combines fuzzy systems and Radial Basis Function (RBF) was proposed. The concept of fusion of multiple classifiers dedicated to specific feature parameters with an exactness of $94.28 \%$ however RBF was portrayed by a quick preparing rate than fuzzy. It removed both texture and statistical features.

(e) MRI Brain Tumor Analysis: For the MRI brain tumor images a general regression neural network (GRNN) based automatic three-dimensional classification method was proposed. This method had good time-consuming rate and classification accuracy. Another intelligent classification technique proposed was Least Squares Support Vector Machines (LS-SVM). It identifies normal and abnormal slices of brain MRI data. This technique had a higher accuracy of classification over other classifiers as the false negative in LS- SVM was very low compared. Due to 
automatic defects detection in MR images of brain, extensive research is being performed.

- Application of Artificial Intelligence in Accounting Databases: The use of artificial intelligence is investigated as the basis to mitigate the problems of accounting databases. The following are some difficulties with existing accounting database systems. The needs of decision makers are not met by accounting information. Humans do not understand or cannot process the computerized accounting databases. Systems are not easy to use. There is focus on the numeric data. Incorporating intelligent systems with accounting databases can assist (either with the leader or autonomous of chief) in the examination of expansive volumes of information with or without coordinate support of the leader. In this way, the systems can examine the data and assist the users understanding or interpreting transactions to determine what accounting events are captured by the system. With the artificial intelligence we store and retrieve knowledge in natural language. There are some artificial intelligence tools or techniques that help in the broader understanding of events captured by the accounting system. There is more emphasis on symbolic or text data rather than just numeric data to capture context. The artificial intelligence and expert system incorporates insight with the database to help users. Without users direct participation such models help the users by sorting through large quantities of data. Such models also assist the decision makers under time constraints; suggest alternatives in the searching and evaluation of data.

- Application of Artificial Intelligence Techniques in the Computer Games: Playing games is one of the most popular uses for computer technology. In the advancement of computer games, they have developed from modest text based to the three-dimensional graphical games with complex and extensive universes. The systems as graphics rendering, playing audio, user input and game artificial intelligence (AI) when assembled give the normal stimulation and make an advantageous computer game. Artificial intelligence is the most essential piece of every computer game and playing the game without artificial intelligence would not be any good times! If we remove artificial intelligence from computer games, the games will be so simple that nobody will be interested in playing the computer games anymore! Without the game AI, the winning would not be difficult at all. Artificial intelligence is used to solve common problems in the computer games and provide the features to the games. In particular, non-playing character (NPC) way discovering, basic leadership and learning are inspected. There are a few different ways that AI adds to present day computer games. Most prominently are unit development, mimicked discernment, circumstance investigation, spatial thinking, learning, bunch coordination, asset portion, directing, rushing, target choice, thus some more. Even context dependent animation and audio use AI.

- Computer Game Problems Solved with AI: Artificial intelligence solves the three common problems: non-playing character (NPC) movement, NPC decision making, and NPC learning. The four artificial intelligence techniques used are Path Finding, Bayesian Networks, Fuzzy Logic, and Genetic Algorithms which help a computer game provide nonplaying character path finding and decision making as well as learning. 


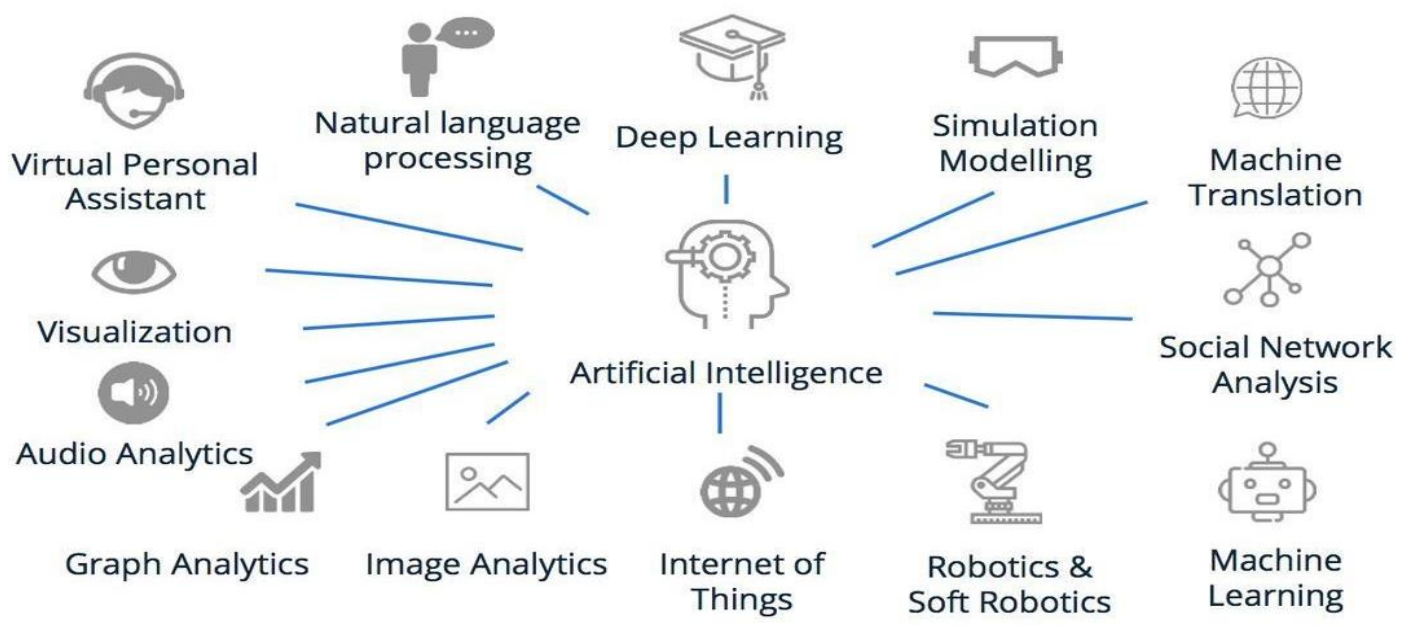

Figure 4: Applications of Artificial Intelligence

\section{Analysis and Findings}

\section{Application of Artificial Intelligence Techniques in Traffic Signal}

(a) Artificial Neural Nets in Transportation: As per decent variety of AI and to its developing utilization I am just ready to depict in this article neural systems use in a few zones of street transport. Nowadays IT era force us to handle more and more information in very short time. That is why it is inevitable to build and utilize such specialized gadgets which can deal with critical data from amount and as indicated by its plan reasonable answer for current circumstance, maybe even anticipate following circumstance. These complicated issues are mostly solved by neural networks utilizing information about data organizing and organization in human mind.

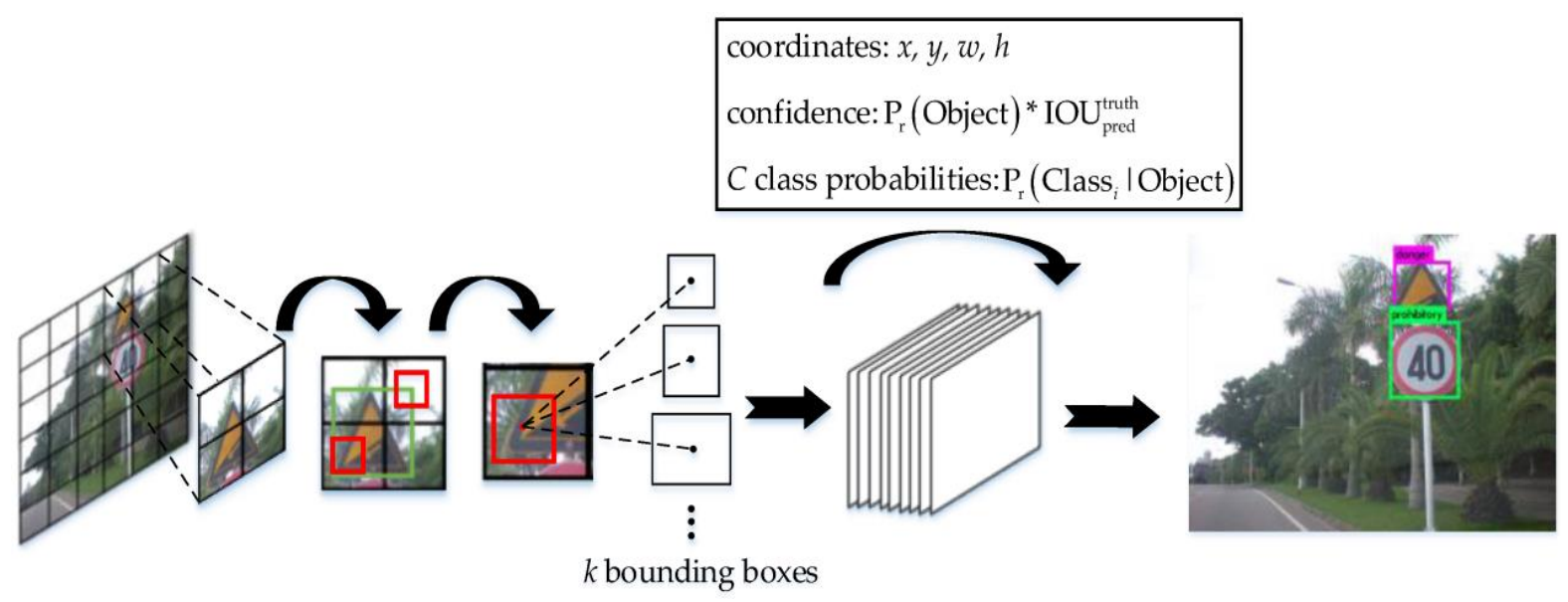

Figure 5: Algorithm of Neural network for traffic control

(b) Traffic Sign Recognition: There are devices that can distinguish, perceive and follow traffic signs from moving vehicle. Recognition is done by colour segmentation and neural nets classification. Existing systems cannot just perceive traffic signs yet in addition locate 
and gather them. Locating is realised by approximating location from GPS device and location of traffic sign acquired from video cam or video file. Traffic signs gathering assist to build traffic sign database which in the similar time creates training data set. Traffic signs are described by features from which the most vital for identifying and perceiving are shading and shape. Detection is done by classical methods based on tresholding and colour segmentation using different colour models or shape models (in black and white images) or their combination. 3D modelling is also often used. There are more methods which use machine learning algorithms for classification and detection.

Traffic signal is a measure of traffic command, which distributes right of way to the traffic flow in time domain. Through comparative study between RBF network and BP network, this paper has selected RBF network to control the traffic single. Green Light Interval is decided by the measurement parameters of detector in the actuated signal control. In the usually, the time phase number of the system is set beforehand. When there is not demand for the vehicles passing in the direction of red light, the green light phase works continually. The system begins the control model until there is interest for the vehicles going towards in the direction of builds the model base on radial basis function, recreates in MATLAB and make an analysis of the reproduced results.

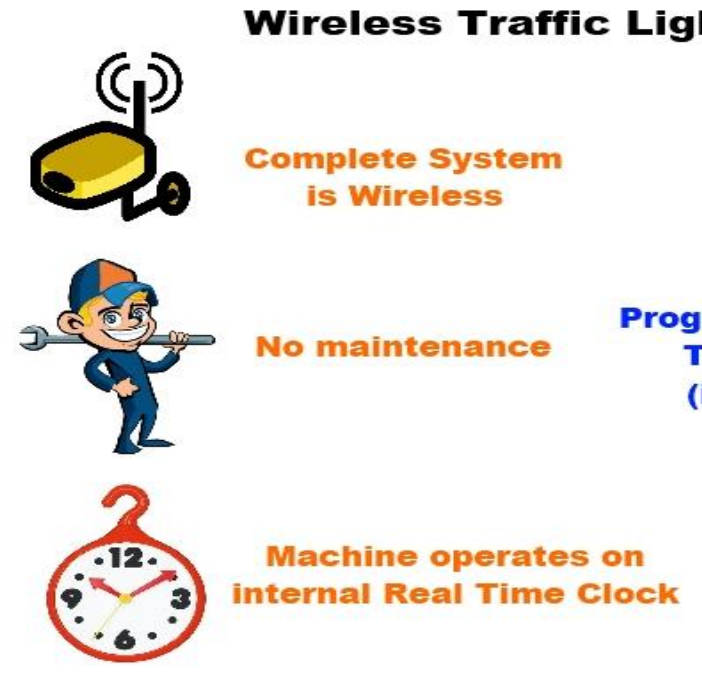

Programmable Automatic

Timing Sequences

(including Flasher)

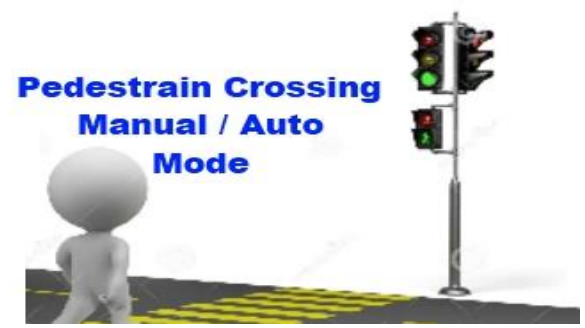

Figure 6: Wireless Traffic light controller

\section{Limitations and Future Scope}

- Using the same techniques of Artificial Intelligence, it would be possible to control the traffic on the road and to completely reducing the amount of accidents happened on road by applying more algorithms.

- In future, the research work can also be carried out using the same techniques, by exploring some other applications of AI.

- In the future enhancement we can make the additional changes in AI that will make traffic better by reducing intersection accidents, reducing traffic in some places as we can assign some value to persons which are crossing the road and the lights red green and orange. 


\section{Conclusion}

The field of artificial intelligence gives the ability to the machines to think analytically, using concepts. Tremendous contribution to the various areas has been made by the Artificial Intelligence techniques from the last 2 decades. Artificial Intelligence will continue to play an increasingly important role in the various fields. This paper is based on the concept of artificial intelligence, areas of artificial intelligence and the artificial intelligence techniques used in the Network Intrusion Detection to protect the network from intruders, in the medical area in the field of medicine, for medical image classification, in the accounting databases, and described how these AI techniques are used in computer games to solve the common problems and to provide features to the games so as to have fun and how to control the traffic and reducing accidents on road. There is bright future in the analysis of Network Intrusion Detection. We conclude that further research in this area can be done as there are very promising and profitable results that are obtainable from such techniques. While scientists have not yet realized the full potential and ability of artificial intelligence. This technology and its applications will likely have far-reaching effects on human life in the years to come.

\section{Acknowledgement}

I would like to express my very great appreciation to Ms. Deepali Shah, Professor, Mumbai University, for her valuable and constructive suggestions and overall help in conducting this research.

\section{References}

[1] Akiyama, T., Sasaki,T.: A neural network approach to the identification of real time origindestination flows from traffic counts. In: Proc. Int. Conf. on Artificial Intelligence Applications in Transportation Engineering (1992).

[2] http://www.akri.org/ai/defs.htm

[3] http://www.dacs.dtic.mil/techs/neural/neural_ToC.html

[4] International Journal of Engineering and Innovative Technology (IJEIT) Volume 4, Issue 10, April 2015

[5] A Review on Artificial Intelligence (International Journal of science and research)

*Corresponding author.

E-mail address: kamble.sony94@ gmail.com 